\title{
Is There Always a Need for Permanent Pacemaker Replacement After Device Infection? A Tale of Two Patients
}

\author{
Munish Sharma $^{\mathrm{a}, \mathrm{d}}$, Daniel A.N. Mascarenhas ${ }^{\mathrm{b}, \mathrm{c}}$
}

\begin{abstract}
There has been an increase in number of cardiac implantable electronic devices (CIEDs) implantation and with this we have witnessed increased rates of CIED infection mainly in elderly population. It is important to assess conditions that may not reliably improve with cardiac pacing or those who lack adequate beneficial effect from permanent pacing before contemplating implantation or reimplantation of these devices. Sometimes, the initial cardiac pathology may revert obviating the need for reimplantation as in the two cases that we have discussed below. This reduces the chance of further infection of CIED, and decreases mortality and morbidity due to recurrent CIED infection and decreases cost of care.
\end{abstract}

Keywords: Cardiac implantable electronic devices; Permanent pacemaker; Device infection; Device reimplantation

\section{Introduction}

Cardiac implantable electronic devices (CIEDs) that include permanent pacemaker (PPM), implantable cardioverter defibrillator (ICD) and cardiac resynchronization therapy (CRT) have become increasingly popular in the management of cardiac diseases over the last few decades in the United States. The implantation rates for PPM and ICD increased by $19 \%$ and 60\% respectively in the United States between 1997 and 2004 [1]. It has been found that more than $70 \%$ of CIED recipients are 65 years of age or older and around $75 \%$ of them have more than one coexisting illnesses as revealed by populationbased surveys in Olmsted County, Minnesota [2,3]. Thus, the increased rates of CIED implantation mainly in elderly population who have more than one comorbid conditions have subsequently increased the rates of CIED infection. There was a $124 \%$ increase in the rate of CIED infection among Medicare

Manuscript submitted December 16, 2017, accepted January 4, 2018

aDepartment of Internal Medicine, Easton Hospital, Easton, PA, USA

${ }^{b}$ Drexel University College of Medicine, Philadelphia, PA, USA

'Easton Hospital, Easton, PA, USA

${ }^{\mathrm{d} C}$ Corresponding Author: Munish Sharma, Department of Internal Medicine,

Easton Hospital, Easton, PA 18042, USA. Email: munishs1@hotmail.com

doi: https://doi.org/10.14740/cr657w beneficiaries from 1990 to 1999 [4]. Thus, it is important to assess and rule out conditions that may not reliably improve with cardiac pacing or those that lack adequate beneficial effect from permanent pacing. There may be conditions, which may revert, and the cardiac pacing may not be permanently needed as evident in our case.

\section{Case Report}

\section{Case 1}

A 93-year-old male with a history of hypertension, coronary artery disease status post a PPM implantation 20 years ago with generator replacements in 2007 and in January 2017 presented 1 month later with erosion at the site of the PPM implantation with serosanguinous drainage from the site in the left side of the chest wall. He had no fever or chills, chest pain, shortness of breath, traumatic injury to the area. The patient was hospitalized on intravenous antibiotics and the decision was made to explant the PPM without immediate reimplantation given that his pacemaker was interrogated before the procedure revealing second-degree type I heart block (Wenckebach). His first PPM was placed after stent placement across a septal perforator resulting in complete atrioventricular (AV) nodal blockade $24 \mathrm{~h}$ post-procedure. The patient underwent explanation of the device and was continued on parenteral antibiotics with the wound site packed with iodoform. He was maintained on telemetry monitoring for 7 days and close observation during the remainder of his hospital course due to the fact that he no longer had the pacemaker implanted for the first time in 20 years. His 12-lead electrocardiography (EKG) during hospitalization revealed trifascicular block with a ventricular rate in the 70s (Fig. 1), hence the decision was made to hold off on the PPM reimplantation. Following completion of intravenous antibiotic therapy for 6 weeks, implantable cardiac monitor (ICM) was implanted to evaluate for the need for PPM in the future. Upon follow-up 6 months later, the patient's ICM interrogation did not reveal any symptomatic bradycardia or pauses that would necessitate PPM reimplantation (Fig. 2).

\section{Case 2}

A 91-year-old gentleman was seen by the cardiologist in India 


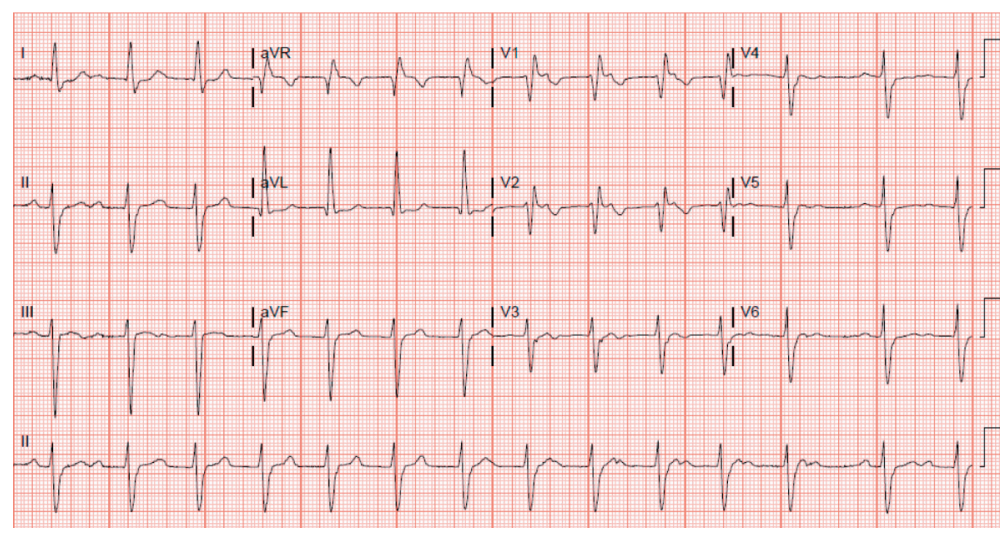

Figure 1. EKG showing trifasicular block.

for a pacemaker that was at the end of life hence a new generator was placed in January 2016. His postoperative course was uncomplicated and did well until May 2017 when he was found to have oozing at the pacemaker site (Fig. 3). In view of the same, the pacemaker was explanted and the subcutaneous tissue was packed with iodoform dressing with complete resolution of subcutaneous infection. Since May 2017, he has been without any symptoms but has been in intermittent atrial fibrillation without any long pauses. A recent Holter monitor revealed no long pauses and showed atrial fibrillation (Fig. 4). The patient was recently diagnosed with lung cancer in the left upper lobe and is being treated for the same with palliative care.

\section{Discussion}

CIED infection poses clinical challenge for the physicians. It also increases mortality, morbidity and financial cost of treatment. The mortality rates due to endocarditis resulting from cardiac device infections range from $31 \%$ to $66 \%$ if the device is not removed [5]. On the contrary, there are several wellreported life-threatening complications associated with device removal. Some of these include tricuspid valve damage, sub-

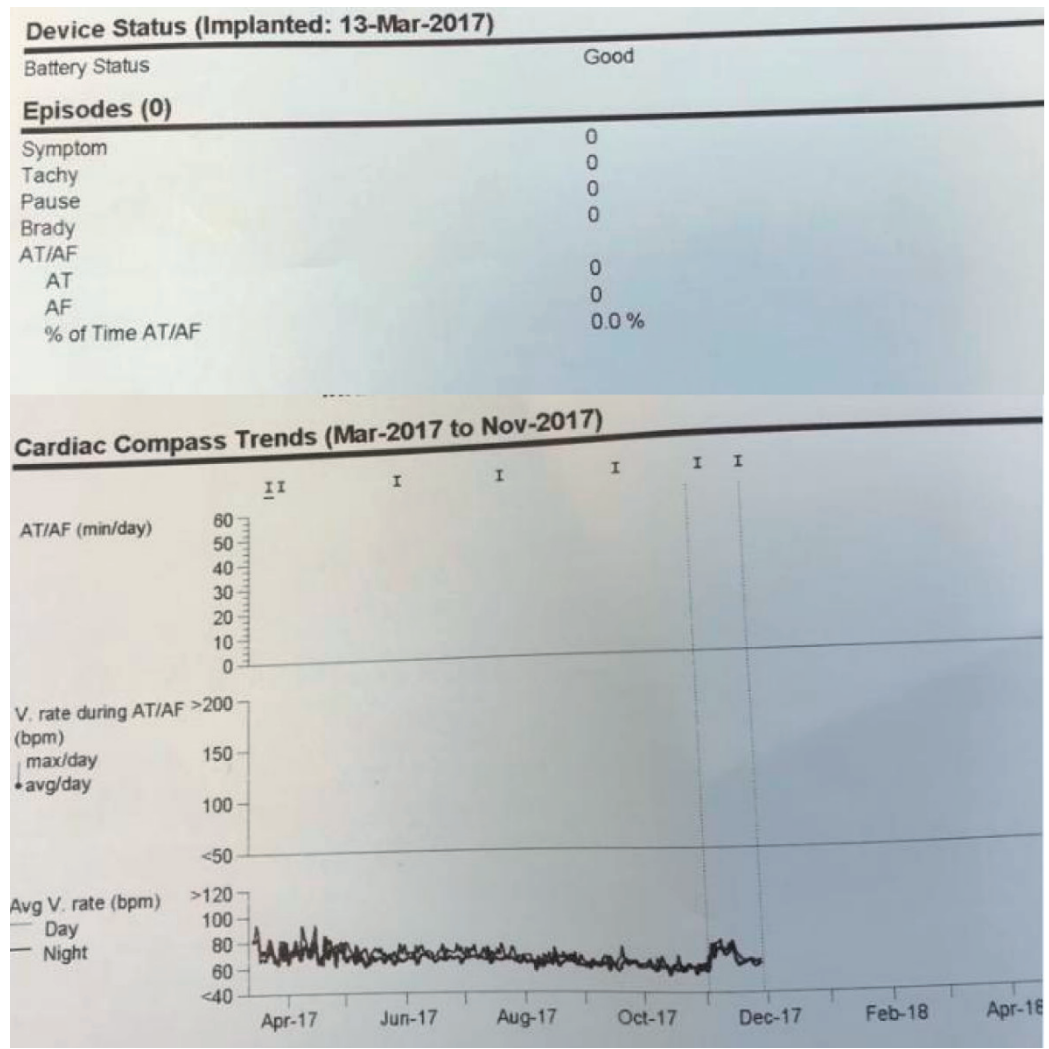

Figure 2. Loop recorder did not reveal any episodes of symptomatic bradycardia or pauses. 


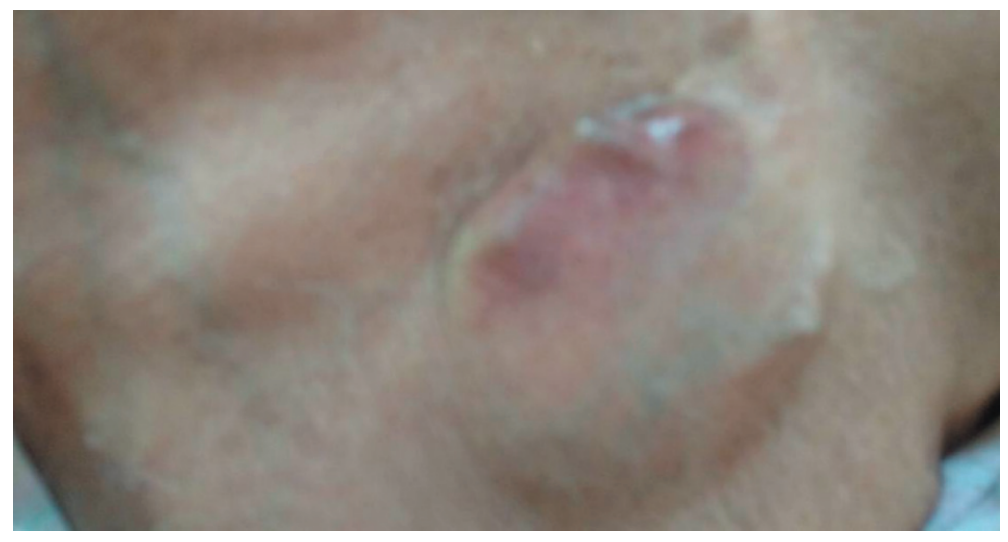

Figure 3. Pacemaker site infection.

clavian vein laceration, hemothorax, pocket hematoma, postoperative cardiac arrest amongst others [4]. Average cost of combined medical and surgical treatment of an infected PPM and ICD ranges from $\$ 25,000$ to $\$ 50,000$ respectively [6].

Though CIED removal is not required for superficial or incisional infection at the pocket site if there is no involvement of the device, complete removal is the standard of treatment for established CIED infections [7]. Adjunctive antimicrobial therapy should be empirically started to cover staphylococcal strains but the final selection of appropriate antimicrobial agent should be based on culture and susceptibility testing. There are no clinical trial data that define the optimal duration of antimicrobial therapy for CIED infection but the general consensus is to treat for 7 - 10 days after device removal if the presentation is device erosion without inflammatory changes; otherwise, 10 - 14 days is considered optimal. In patients with positive blood cultures $>24 \mathrm{~h}$ despite CIED removal and appropriate antimicrobial therapy, parenteral therapy for at least 4 weeks should be given, even if transesophageal echocardiography (TEE) is negative for valvular vegetations [8].

It is essential to evaluate a patient with extraction of infected CIED for new device placement but it should also be kept in mind that around one third to one half of such patients may not require new CIED placement [4]. Reversal of initial cardiac pathology that required CIED implantation, lack of appropriate initial clinical indication, refusal of patient to get new device are few scenarios which obviate the need for new CIED placement and this can help avoid new device infection. With lack of definite prospective trial examining timing of new device replacement and risk of relapsing infection; there is no uniform consensus on optimal timing of device replacement. Several investigators have recommended waiting for the blood cultures to be negative before a new device can be placed [9].

Conditions with a lack of adequate evidence of benefit from permanent pacing like syncope of unknown cause, asymptomatic sinus bradycardia, asymptomatic first degree and second degree Mobitz I (Wenckebach) AV block, long QT syndrome or torsades de pointes due to a reversible cause should be evaluated carefully before implanting CIED. In case of reversal of pathology that initially required CIED, it is advisable to avoid reimplantation. A continuous monitoring for future need of the PPM can be done by alternative less invasive device like ICM.

\section{Conclusions}

It is imperative to evaluate a patient carefully before considering reimplantation of a PPM after its removal due to infection. The initial cardiac pathology may revert obviating the need for reimplantation. This reduces the chance for further infection of CIED, decreases mortality and morbidity due to recurrent

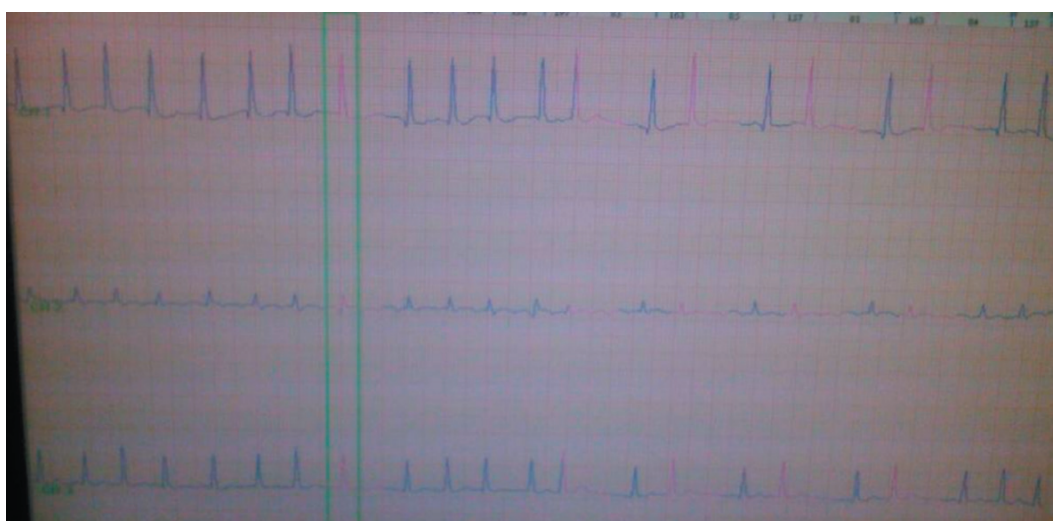

Figure 4. Holter monitor showing atrial fibrillation. 
CIED infection and decreases cost of care. Less invasive continuous monitoring device like ICM can be an effective alternative to assess the patient for further consideration for CIED reimplantation.

\section{Conflict of Interest}

The authors declare no conflict of interest.

\section{References}

1. Zhan C, Baine WB, Sedrakyan A, Steiner C. Cardiac device implantation in the United States from 1997 through 2004: a population-based analysis. J Gen Intern Med. 2008;23(Suppl 1):13-19.

2. Uslan DZ, Tleyjeh IM, Baddour LM, Friedman PA, Jenkins SM, St Sauver JL, Hayes DL. Temporal trends in permanent pacemaker implantation: a population-based study. Am Heart J. 2008;155(5):896-903.

3. Lin G, Meverden RA, Hodge DO, Uslan DZ, Hayes DL, Brady PA. Age and gender trends in implantable cardioverter defibrillator utilization: a population based study. J Interv Card Electrophysiol. 2008;22(1):65-70.

4. Sohail MR, Uslan DZ, Khan AH, Friedman PA, Hayes
DL, Wilson WR, Steckelberg JM, et al. Management and outcome of permanent pacemaker and implantable cardioverter-defibrillator infections. J Am Coll Cardiol. 2007;49(18):1851-1859.

5. Cacoub P, Leprince P, Nataf P, Hausfater P, Dorent R, Wechsler B, Bors V, et al. Pacemaker infective endocarditis. Am J Cardiol. 1998;82(4):480-484.

6. Ferguson TB, Jr., Ferguson CL, Crites K, CrimminsReda P. The additional hospital costs generated in the management of complications of pacemaker and defibrillator implantations. J Thorac Cardiovasc Surg. 1996;111(4):742-751; discussion 751-742.

7. Chua JD, Wilkoff BL, Lee I, Juratli N, Longworth DL, Gordon SM. Diagnosis and management of infections involving implantable electrophysiologic cardiac devices. Ann Intern Med. 2000;133(8):604-608.

8. Baddour LM, Epstein AE, Erickson CC, Knight BP, Levison ME, Lockhart PB, Masoudi FA, et al. Update on cardiovascular implantable electronic device infections and their management: a scientific statement from the American Heart Association. Circulation. 2010;121(3):458477.

9. Trappe HJ, Pfitzner P, Klein H, Wenzlaff P. Infections after cardioverter-defibrillator implantation: observations in 335 patients over 10 years. Br Heart J. 1995;73(1):2024. 